\title{
Ballistic Electrons in Ferromagnet/Semiconductor Hybrid Structures: From Nanomagnetometry to Spin Injection
}

\author{
D. GRUNDLER* \\ Institut für Angewandte Physik und Zentrum für Mikrostrukturforschung \\ Universität Hamburg, Jungiusstr. 11, 20355 Hamburg, Germany
}

\begin{abstract}
We review our recent experimental and theoretical studies on ferromagnet/semiconductor hybrid structures and discuss the role of ballistic electrons in such systems. We focus in particular on two peculiar features: first, ballistic electrons in semiconductors are shown to be in particular sensitive to local details of an inhomogeneous stray field. We argue that this can preferentially be used for nanomagnetometry. Second, we show theoretically that, in case of the injection of ballistic electrons from a metallic ferromagnet into a semiconductor, a spin-dependent interface resistance arises due to band-structure mismatch that causes spin filtering at the interface. Recent band-structure calculations suggest that for an epitaxial interface a nearly $100 \%$ spin-polarized current might be generated in a spin-injection experiment.
\end{abstract}

PACS numbers: 72.25.Hg, 72.25.Mk, 72.25.Dc, 73.23.Ad, 73.40.Sx, 75.60.Ej

\section{Introduction}

Ferromagnet(fm)/semiconductor(sc) hybrid structures are nowadays of interest both for basic research in solid state physics and for applications in magnetoelectronics and spintronics [1,2]. Magnetic properties of nanostructured ferromagnets are currently a research field of great interest [3-7] and are important for nonvolatile magnetic storage [8] and for spin-injecting sources and spin-detecting

*e-mail: grundler@physnet.uni-hamburg.de 
drains in spin-transport devices [9]. In the latter kind of devices, the spin of the electron might be used as the information rather than the electronic charge. By this means, intriguing new perspectives are arising. If, e.g., magnetic and semiconducting materials were combined in hybrid structures, researchers and engineers in the microelectronics business might be able to realize multifunctional devices which combine both, nonvolatile data storage and ultrafast data processing. In addition, the prospect would be a low power consumption. The spin itself as a genuine two-level quantum system can also serve as a qubit. In case of coherent spin transport in semiconductors one might therefore also think about such visionary concepts like solid-state implementations of quantum computation.

In this paper, we review our recent work concerning ballistic electrons in ferromagnet/semiconductor hybrid structures. We focus on two important issues: (i) the effect of stray fields originating from ferromagnetic contacts and acting on the orbital motion of charge carriers in the semiconductor [10], and (ii) the effect of the spin-dependent interface resistance between a ferromagnetic metal and a III-V semiconductor. The latter is shown to give rise to the injection of a spin-polarized current via a process which is called spin filtering [11-13]. The results presented here are discussed in the framework of the spin field-effect transistor (spin FET) which was theoretically proposed by Datta and Das [9] in 1990 (Fig. 1).

The paper is organized as follows: in Sec. 2.1 we introduce in brevity the Landauer-Büttiker formalism which is the common theory in mesoscopic physics to describe the transport of ballistic electrons. In Sec. 2.2 we discuss stray-field effects, in Sec. 2.3 we outline the idea of spin filtering.

\section{Ballistic electrons in ferromagnet/semiconductor hybrid structures}

In the spin FET as originally proposed by Datta and Das [9], the ballistic and coherent transport of spin-polarized electrons is assumed to occur in a semiconductor heterostructure which exhibits the Rashba effect [14]. The latter is the spin-orbit interaction that can be tuned and controlled via an electric field in a

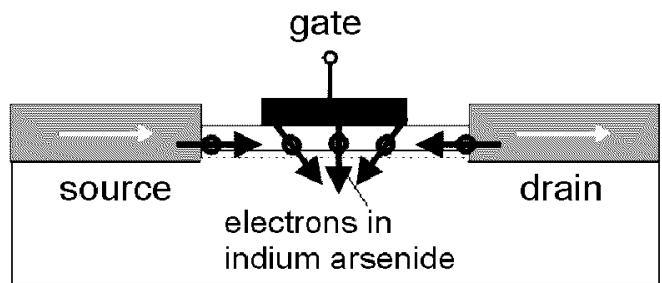

Fig. 1. Schematic side view on the spin field-effect transistor proposed by Datta and Das (after [9]). Source and drain are ferromagnetic contacts, the white arrows indicate the direction of the magnetization. Ballistic and coherent spin-polarized electrons undergo a spin precession along the channel in case of spin-orbit interaction (Rashba effect). 
compound semiconductor lacking inversion symmetry [15-18]. In Fig. 1, a gate electrode on top of a modulation-doped III-V semiconductor heterostructure is assumed to control the electric field perpendicular to the plane of the electron system, and by this means to vary the spin-orbit interaction which causes spins to precess. Datta and Das have predicted intriguing new spin transport phenomena: as a function of gate voltage, the electron spins should change their orientation along the semiconductor channel. Using this effect, one could tune the magnetoresistance by an electrical field. The pioneering theoretical work was based on a one-dimensional electron system between the ferromagnetic source and drain. Recent theoretical studies [19] and experimental results [20, 21] suggest that a mesoscopic two-dimensional electron system (2DES), that exhibits a lateral width on the micrometer length scale, might also be suitable for studying spin-transport phenomena of ballistic electrons in a spin FET configuration.

\subsection{Transport theory: Landauer-Büttiker formalism}

In the following we outline the Landauer-Büttiker formalism which is the well-accepted transport theory for ballistic electrons in mesoscopic physics. In case of a diffusive two-dimensional electron system the conductivity would be given by $\sigma=n_{\mathrm{s}} e \mu$, where $n_{\mathrm{s}}$ is the carrier density and $\mu$ is the mobility. From this one can calculate the conductance $G$ from $G=(W / L) \sigma$, where $W$ is the width and $L$ is the length of the device. Deviations from the diffusive picture are expected to occur if the lateral sizes $W$ and $L$ are smaller than the electron mean free path $l_{e}$. Then the ballistic transport regime may be entered where the Landauer-Büttiker transport theory is well established. It suggests that, instead of being scattered as particles, the electrons are transmitted and reflected like waves travelling through the device. For example, in case of a constriction the two-terminal conductance is given by

$$
G=\left(e^{2} / h\right) M T,
$$

where $M$ denotes the number of spin-nondegenerate modes (in a mesoscopic 2DES $\left.M=2 \times \operatorname{Int}\left[k_{\mathrm{F}} W / \pi\right]\right)$ and $T \leq 1$ denotes the transmission coefficient. A comprehensive overview and detailed discussion on the Landauer-Büttiker formalism and its application can be found in Refs. [22, 25]. The multi-terminal resistance is calculated on the basis of quantum-mechanically derived transmission coefficients $T_{m n}$. In case of a configuration like the one in the inset of Fig. 3a which will be discussed in the next section the resistance $R_{24,13}$ reads [24]

$$
R_{24,13}=\frac{h}{e^{2}}\left(T_{24} T_{31}-T_{21} T_{34}\right) / D
$$

where the numbers label leads. The parameter $D$ is positive and constant for a given device. The intriguing result of Eq. (2) is that a negative resistance is expected if the current is applied around a bend and if ballistic electrons are present. This is due to the transmission coefficients $T_{21}$ and $T_{34}$ which are larger than $T_{24}$ and $T_{31}$. 
In the latter case electrons have to turn round a corner which is an unlikely process for electrons with a large forward momentum $k$. The negative resistance was observed by Takagaki et al. in 1988 [26] on a micron-sized 2DES at low temperature. They also showed that the absolute value of the bend resistance was reduced by applying a homogeneous magnetic field perpendicular to the mesoscopic 2DES. According to Eq. (2) one could argue that the transmission coefficients $T_{21}$ and $T_{34}$ were reduced due to the Lorentz force acting on the electrons.

\subsection{Stray-field effect on ballistic electrons}

In the following we demonstrate that ballistic electrons in a mesoscopic 2DES are extremely sensitive to an inhomogeneous magnetic field [10]. For our experiments, we fabricate 2DES cross-junctions with a lateral size which is smaller than the electron mean free path and integrate permalloy $(\mathrm{Py})$, i.e., $\mathrm{Ni}_{80} \mathrm{Fe}_{20}$, micromagnets on top. By this means, we are able to generate characteristic stray-field patterns within the junction region by changing the domain configuration of the micromagnets by an in-plane magnetic field. Here we discuss our results obtained on a cross-junction with a two-micromagnet system on top (Fig. 2). The latter is one of the favored systems for spin-injection/spin-detection contact configurations in a lateral spin-transport device with sub- $\mu$ m channel length [27-29].

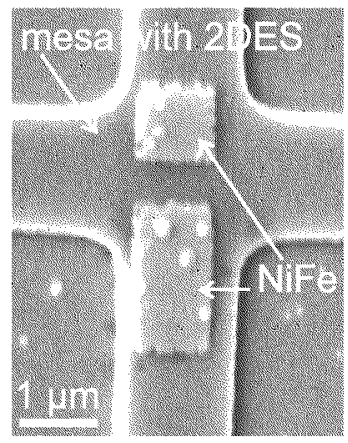

Fig. 2. Ferromagnet/semiconductor hybrid structure for studying stray-field effects. The etched mesa contains the mesoscopic 2DES $90 \mathrm{~nm}$ below the surface. The cap layer of the heterostructure is insulating. The ferromagnetic material is permalloy (labelled $\mathrm{NiFe}$ ).

Micron-sized cross-junctions have been fabricated in a $\mathrm{GaAs} / \mathrm{Al}_{0.3} \mathrm{Ga}_{0.7} \mathrm{As}$ heterostructure using electron-beam lithography and photolithography followed by a wet etching process. The electronic width of the devices is $W=1.7 \mu \mathrm{m}$. Contacts are fabricated using evaporation and lift-off technique. The 2DES is $90 \mathrm{~nm}$ below the surface with a carrier density of $n_{\mathrm{s}}=2.7 \times 10^{11} \mathrm{~cm}^{-2}$ and a mobility of $\mu=3.5 \times 10^{5} \mathrm{~cm}^{2} /(\mathrm{V} \mathrm{s})$. All experiments are performed at the temperature of $T=4.2 \mathrm{~K}$. Then the electron mean free path is calculated to be $l_{\mathrm{e}}=3 \mu \mathrm{m}$. Hence, 
the device is operated in the ballistic regime. The number of ballistic modes is $M=70$.

Polycrystalline Py micromagnets are integrated using electron beam lithography, evaporation, and lift-off techniques. They have been capped by a $9 \mathrm{~nm}$ gold layer. To monitor the effect of local fields on the ballistic electrons bend- and transfer-resistance measurements $[23,24,26]$ were performed in a ${ }^{4} \mathrm{He}$ cryostat with a superconducting magnet at $4.2 \mathrm{~K}$. The external magnetic field was in the plane of the 2DES and along the easy axis of the longer micromagnet. The applied field was swept in small steps of a few tenth of a mT. The current $I$ of $3 \mu \mathrm{A}$ injected in the Hall bar was modulated at $37 \mathrm{~Hz}$.

Bend-resistance curves are shown in Fig. 3 measured on the cross-junction containing a two-micromagnet system with a separation $d=200 \mathrm{~nm}$. In Fig. 3a the current is injected at the injector lead 2 with respect to lead 4 and the voltage is measured between leads 1 and 3. By this means, electrons which transverse the cross-junction ballistically are measured at detector probe 1 [26]. The bend resistance $R_{24,13}$ exhibits a hysteretic "butterfly"-like shape which is reproducible in several runs and cooling cycles. Broken vertical lines and large letters on the top indicate in-plane fields where large variations (jumps) are observed in the resistance trace. These are interpreted to be due to irreversible changes in the domain

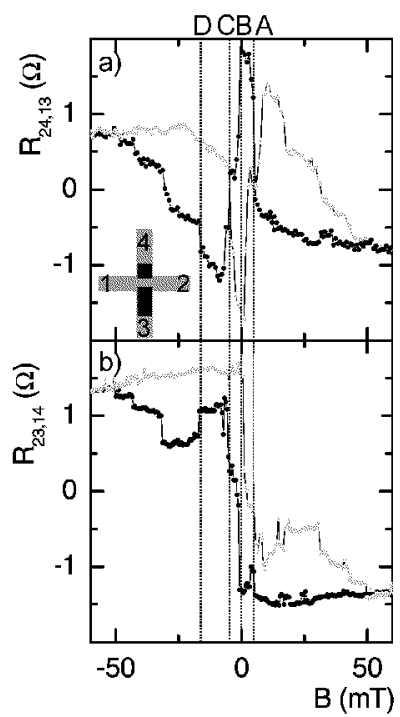

Fig. 3. Measurements of the bend resistance in two different configurations in an in-plane magnetic field $B$. The black traces are measured from positive to negative fields, the gray traces vice versa. Vertical lines indicate irreversible jumps observed in the black traces. A negative offset value has been subtracted from the data for clarity. The leads are labelled in the inset of part (a) [30]. 


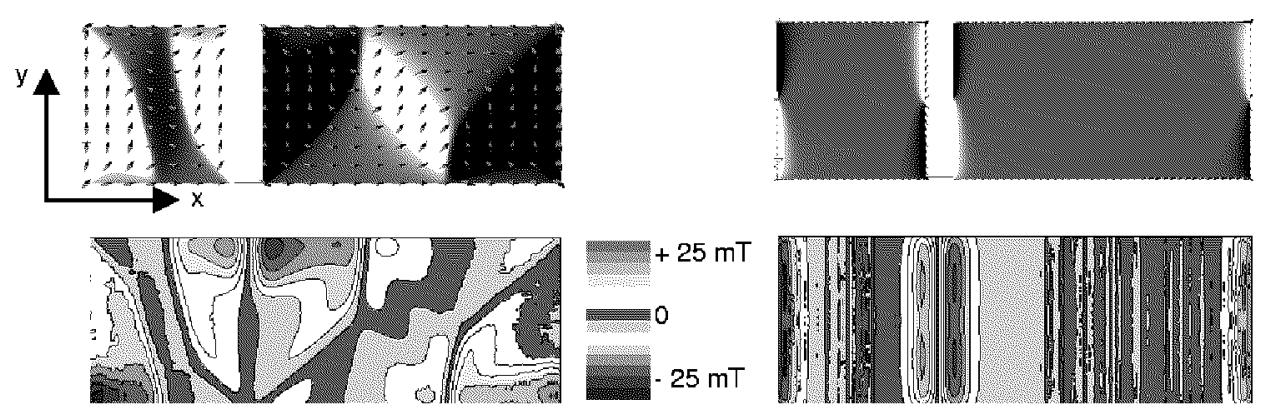

Fig. 4. Simulated domain configuration and stray-field component $90 \mathrm{~nm}$ below the Py two-micromagnet system at $B_{x}=-3.45 \mathrm{mT}$ (left) and $B_{x}=-78 \mathrm{mT}$ [32]. Tiny arrows and the background gray-scale color plot in the upper panel represent the orientation of the microscopic magnetic moments.

configuration ${ }^{\dagger}$ which in turn lead to variations in the magnetic-field pattern in the mesoscopic 2DES. In Fig. 4 we have calculated the perpendicular component of the stray field $90 \mathrm{~nm}$ underneath the two-micromagnet system.

On the basis of Eq. (2) and Fig. 4 we argue that, locally, strong Lorentz forces act on the electrons in such a way that transmission coefficients are changed. As a result, part of the ballistic electrons are deflected. This seems to depend critically on details of the stray-field pattern. Strikingly, we find that this effect is very sensitive to the lead configuration: in Fig. $3 \mathrm{~b}$ we have measured $R_{23,14}$ by interchanging the leads. Now we observe a different shape of the hysteretic resistance trace. By changing current and voltage leads we have obviously probed a different region. Very intriguingly, irreversible jumps, though different in sign and in height, are observed at field positions which agree well with positions of jumps found in Fig. 3a. This underlines that they originate from characteristic changes in the domain configuration of the two-micromagnet system. The ballistic electrons are scattered at small spots of strong magnetic field [31].

We have also performed transfer-resistance measurements on our hybrid structures. This means that the current leads and the voltage probes are not at one and the same cross-junction. In our case, the current was injected around a bend that was $10.5 \mu \mathrm{m}$ apart from the cross containing the two-micromagnet system, i.e., the lateral distance between injector and detector was larger than the mean free path $l_{e}=3 \mu \mathrm{m}$. In Fig. 5 we show the hysteretic behavior of the measured resistance. The peak-to-peak value in the resistance trace has decreased by a factor of about 20 if compared to Fig. 3 and jumps are diminished. Nevertheless, even at this large distance, which is three times the mean free path $l_{e}$, stray-field effects on the ballistic electrons can still be monitored. Our results are much more important since nonlocal resistance measurements have been intro-

${ }^{\dagger}$ A detailed discussion on the supposed domain configurations of the two-micromagnet system in an applied field can be found in Ref. [10, 29, 30]. 
duced recently to study spin-transport phenomena in ferromagnet/semiconductor hybrid structures [33]. As a result, we find that:

(i) Ballistic electrons are very sensitive to local Lorentz forces and are readily deflected. This effect can be used for nanomagnetometry with high spatial resolution. By this means, we have recently investigated the switching of the nanometer-sized end domains (compare Fig. 4b) that occur due to the demagnetizing effect in micromagnets [30].

(ii) In a lateral spin-injection/spin-detection device incorporating a mesoscopic 2DES with Rashba spin-orbit interaction ballistic electrons might exist with curved trajectories due to local Lorentz forces. This could alter the spin-transport behavior.

(iii) Stray-field effects are prominent in a nonlocal measurement configuration (compare Fig. 5) if ballistic electrons can reach the voltage probes.

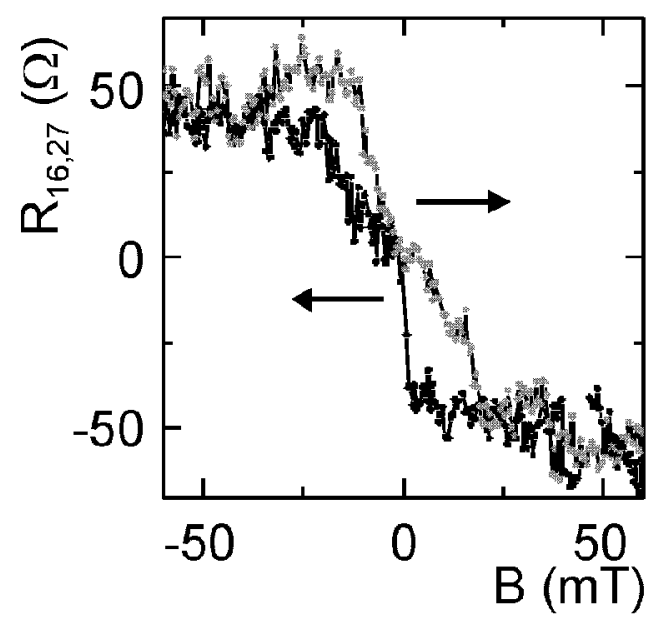

Fig. 5. Measurement of the nonlocal transfer resistance, i.e., the separation between the current injector and the voltage detector is $10.5 \mu \mathrm{m}$. A negative offset value has been subtracted for clarity.

In case of a semiconductor channel between ferromagnetic sources and drains, it is now evident that a detailed knowledge of the domain configuration of the ferromagnetic contacts and, in particular, of the evanescent stray fields is an important prerequisite to interpret the transport of ballistic electrons.

\subsection{Spin filtering of ballistic electrons at the ferromagnet/semiconductor interface}

Currently, there is a lively discussion by which means efficient spin injection into semiconductors is possible if a metallic ferromagnet is used as a spin-injecting source. If bulk conductivities of the two different materials were regarded and a 
spin-dependent interface resistance was neglected, a basic obstacle for spin injection was supposed to exist in the diffusive transport regime [34]. Recently, it was suggested that the situation is significantly improved if a spin-dependent barrier layer $[35-38]$ is incorporated at the interface. This barrier can either be an artificial tunnelling barrier made from an oxide or a Schottky barrier which forms naturally in many cases of a metal/semiconductor contact (e.g. in case of GaAs). Now there seems to be experimental evidence that spin injection of hot electrons via a barrier layer is feasible and efficient [38-41].

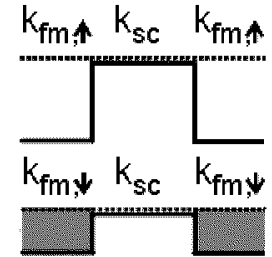

(a)

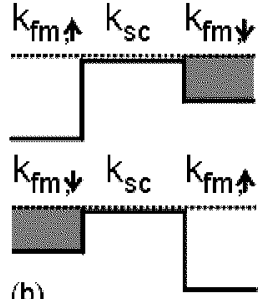

(b)

Fig. 6. Schematic representation of the spin-filtering effect: shown is the bottom conduction band edge and the Fermi energy $E_{\mathrm{F}}$ (dotted horizontal line) in the $\mathrm{fm} / \mathrm{sc} / \mathrm{fm}$ hybrid structure in case of (a) parallel and (b) antiparallel alignment of the magnetization if no Schottky barrier is present. In this scenario, the majority and minority spins of the ferromagnet entering the 2DES as plane waves (the states are split due to the exchange energy) exhibit a different band-structure mismatch, i.e., a different potential step, and obey spin-dependent specular reflection. This results, in general, in different transmission and reflection coefficients and leads to spin filtering at the $\mathrm{fm} / \mathrm{sc}$ interface and to a magnetoresistance effect in a $\mathrm{fm} / \mathrm{sc} / \mathrm{fm}$ hybrid structure.

It has recently been discussed that in the ballistic transport regime efficient spin injection should be possible even without introducing a separate barrier layer [11-13]. The idea is that the band-structure mismatch (Fig. 6) at the interface between a metallic ferromagnet and a semiconductor favors spin filtering which then generates a spin-polarized current. In our approach, we first modelled this effect on the basis of the Landauer-Büttiker formalism (Eq. (1)) for a point contact using the ansatz of plane waves in a free-electron model. In this case, we distinguish between the two transmission coefficients $T_{\uparrow}$ and $T_{\downarrow}$ for majority and minority spins, respectively, which enter the semiconductor from the ferromagnet. In our model, for electrons with a momentum $k$ perpendicular to the interface the coefficients $T$ are calculated from

$$
T=\frac{1}{1+Z^{2}},
$$

with the parameter [43]

$$
Z^{2}=\frac{\left(r_{v}-1\right)^{2}}{4 r_{v}} \geq 0
$$


where the group velocities $v$ enter by $r_{v}=v_{\mathrm{sc}} / v_{\mathrm{fm}}$. In the free-electron model, we take the Fermi velocity $v_{\mathrm{F}}=\hbar k_{\mathrm{F}} / m^{*}$ at the Fermi energy $E_{\mathrm{F}}\left(m^{*}\right.$ is the effective mass of the electron in the material). In the absence of spin-flip scattering, the transmission coefficients are in general different for the two orthogonal spin states leading to spin-dependent specular reflection (Fig. 7). The latter effect is the basic concept of spin filtering via band-structure mismatch. As important results we find that spin filtering:

(i) can take place at the interface between a metallic ferromagnet and a semiconductor and should generate a spin-polarized current,

(ii) should depend on the specific combination of materials due to variations in the band structure and

(iii) should depend on the electron energy, i.e., in our case on $E_{\mathrm{F}}$.

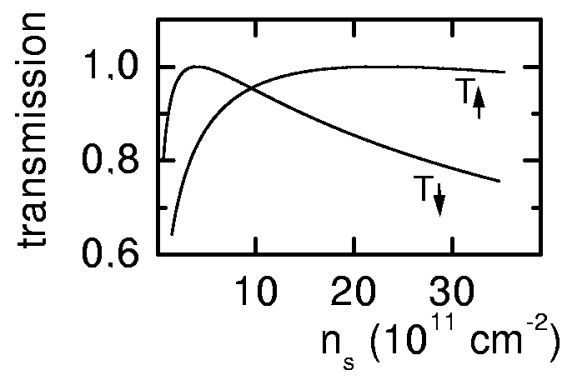

Fig. 7. Spin-dependent transmission probabilities at the interface between a ferromagnet $(\mathrm{fm})$ and a semiconductor (sc) plotted as a function of 2DES carrier density $n_{\mathrm{s}}$ [13]. We assume the momentum $k$ of the electrons to be perpendicular to the interface. The effective mass in the sc is $m_{\mathrm{sc}}^{*}=0.036 m_{\mathrm{e}}$, a reasonable value for electrons in an InAs-based heterostructure. The $\mathrm{fm}$ is Fe with $k_{\mathrm{F}, \uparrow}=1.05 \times 10^{8} / \mathrm{cm}$ and $k_{\mathrm{F}, \downarrow}=0.44 \times 10^{8} / \mathrm{cm} \mathrm{[42]}$. For the metal, we took $m_{\mathrm{fm}}^{*}=m_{\mathrm{e}}$. The value $n_{\mathrm{s}}=35 \times 10^{11} \mathrm{~cm}^{-2}$ corresponds to a Fermi energy $E_{\mathrm{F}}$ of about $0.22 \mathrm{eV}$ in the sc.

Within the free-electron model we find an efficiency of injecting a spin-polarized current of the order of ten per cent. Here, Fe on InAs, that does not form a Schottky barrier, might be one of the favored combinations $[11,13]$. Spin injection persists even if we include oblique angles for the momentum $k$ with respect to the interface. Detailed calculations for a ferromagnet/2DES/ferromagnet hybrid structure with a mesoscopic 2DES in InAs exhibiting spin-orbit interaction can be found in Ref. [19]. The results show that including spin filtering in the Datta-and-Das approach recovers the effect of a gate-voltage modulated magnetoresistance due to spin precession. In addition, for the coherent spin transport Fabry-Perot-like resonances are predicted to occur in the hybrid structures [19].

Very recently, $a b$ initio band-structure calculations $[44,45]$ on the spin filtering effect for epitaxial interfaces have been performed in case of various different material combinations (Table). The main difference is here that (i) the full 
TABLE

Degree of spin polarization in the sc derived on the basis of band structure calculations if an epitaxial interface is assumed to exist between fm and sc. In case of ZnSe and GaAs a Schottky barrier with a width of 144 monolayers is inserted in the calculation [44]. The degree of spin polarization further improves if this width is decreased. The Fermi energy is $10 \mathrm{meV}$ in Ref. [44] and $20 \mathrm{meV}$ in Ref. [45].

\begin{tabular}{c|c|c|c}
\hline \hline Fm/sc hybrid structure & Termination of sc & Degree of spin polarization & Ref. \\
\hline $\mathrm{Fe} / \mathrm{GaAs}(001)$ & $\mathrm{Ga}$ & $99.97 \%$ & {$[44]$} \\
$\mathrm{Fe} / \mathrm{GaAs}(001)$ & $\mathrm{As}$ & $97.6 \%$ & {$[44]$} \\
$\mathrm{Fe} / \mathrm{ZnSe}(001)$ & $\mathrm{Zn}$ & $77 \%$ & {$[44]$} \\
$\mathrm{Fe} / \mathrm{ZnSe}(001)$ & $\mathrm{Se}$ & $99.3 \%$ & {$[44]$} \\
$\mathrm{Fe} / \mathrm{InAs}(001)$ & $\mathrm{In}$ & $98 \%$ & {$[45]$} \\
$\mathrm{Fe} / \mathrm{InAs}(001)$ & $\mathrm{As}$ & $89 \%$ & {$[45]$}
\end{tabular}

Bloch-wave ansatz and the lattice-periodic potentials are included, that (ii) by this means the electron states in the transition metal are far better described and that (iii) the chemical bonds at the interface are considered. The symmetry of the electron wave functions for majority and minority spins impose such a mismatch between the ferromagnet and the semiconductor that the spin filtering is even more prominent (Table) than in the free-electron model of Fig. 7. The energy-dependent transmission coefficients $T_{\uparrow}$ and $T_{\downarrow}$ are now orders of magnitude different for majority and minority spins [44]. Hence, almost ideal spin filtering is expected leading in some cases to a spin polarization near $100 \%$. Table shows that the spin filtering is effective in case of an ohmic fm/sc contact (Fe on InAs) and in case of a Schottky barrier (Fe on ZnSe or GaAs). Band-structure calculations on $\mathrm{fm} / \mathrm{sc} / \mathrm{fm}$ hybrid structures predict very pronounced magnetoresistance effects due to such highly polarized spin currents [46]. Here, again, peculiar Fabry-Perot-like resonances are predicted to occur.

\section{Conclusions}

We have discussed the physics of ballistic electrons in ferromagnet/ semiconductor hybrid structures. They offer intriguing aspects: they are very sensitive to local details of the stray field generated by micromagnets. By this means, spatially resolved studies on the magnetization reversal in micromagnets are feasible. Ballistic electrons might also favorably be used to inject efficiently spin-polarized currents into a semiconductor.

\section{Acknowledgments}

This work was only possible due to excellent cooperations. Gratefully acknowledged are contributions to this work by P.H. Dederichs, D. Heitmann, 
T.M. Hengstmann, Ch. Heyn, C.-M. Hu, N. Klockmann, T. Matsuyama, G. Meier, U. Merkt, H. Rolff and O. Wunnicke. Our work is supported by the DFG via Sonderforschungsbereich SFB 508, via He 1938/9, by the NEDO program on spintronics and by the BMBF via project $13 \mathrm{~N} 8283$.

\section{References}

[1] S.A. Wolf, D.D. Awschalom, R.A. Buhrman, J.M. Daughton, S. von Molnar, M.L. Roukes, A.Y. Chtchelkanova, D.M. Treger, Science 294, 1488 (2001) and references therein.

[2] D. Grundler, Phys. World 15, 39 (2002).

[3] K.J. Kirk, J.N. Chapman, C.D.W. Wilkinson, Appl. Phys. Lett. 71, 539 (1997).

[4] W.J. Gallagher, S.S.P. Parkin, Y. Lu, X.P. Bian, A. Marley, R.A. Altman, S.A. Rishton, K.P. Roche, C. Jahnes, T.M. Shaw, G. Xiao, J. Appl. Phys. 81, 3741 (1997).

[5] R.D. Gomez, T.V. Luu, A.O. Pak, K.J. Kirk, J.N. Chapman, J. Appl. Phys. 85, 6163 (1999).

[6] M. Hwang, M.C. Abraham, T.A. Savas, H.I. Smith, R.J. Ram, C.A. Ross, J. Appl. Phys. 87, 5108 (2000).

[7] R.P. Cowburn, Phys. Rev. B 65, 092409 (2002).

[8] S.S.P. Parkin, K.P. Roche, M.G. Samant, P.M. Rice, R.B. Beyers, R.E. Scheuerlein, E.J. O'Sullivan, S.L. Brown, J. Bucchigano, D.W. Abraham, Y. Lu, M. Rooks, P.L. Trouilloud, R.A. Wanner, W.J. Gallagher, J. Appl. Phys. 85, 5828 (1999).

[9] S. Datta, B. Das, Appl. Phys. Lett. 56, 665 (1990).

[10] D. Grundler, T.M. Hengstmann, N. Klockmann, Ch. Heyn, D. Heitmann, Physica E 12, 248 (2002).

[11] D. Grundler, Phys. Rev. Lett. 86, 1058 (2001).

[12] G. Kirczenow, Phys. Rev. B 63, 054422 (2001).

[13] D. Grundler, Phys. Rev. B 63, R161307 (2001).

[14] Yu.A. Bychkov, E.I. Rashba, J. Phys. C, Solid State Phys. 17, 6039 (1984).

[15] J. Nitta, T. Akazaki, H. Takayanagi, T. Enoki, 1997 Phys. Rev. Lett. 78, 1335 (1997).

[16] C.M. Hu, J. Nitta, T. Akazaki, H. Takayanagi, J. Osaka, P. Pfeffer, W. Zawadzki, Phys. Rev. B 60, 7736 (1999).

[17] T. Matsuyama, R. Kürsten, C. Meissner, U. Merkt, Phys. Rev. B 61, 15588 (2000).

[18] D. Grundler, Phys. Rev. Lett. 84, 6074 (2000).

[19] T. Matsuyama, C.M. Hu, D. Grundler, G. Meier, U. Merkt, Phys. Rev. B 65, $155322(2002)$.

[20] C.M. Hu, J. Nitta, A. Jensen, J.B. Hansen, H. Takayanagi, Phys. Rev. B 63, 125333 (2001).

[21] G. Meier, T. Matsuyama, U. Merkt, Phys. Rev. B 65, 125327 (2002). 
[22] M. Büttiker, Phys. Rev. Lett. 57, 1761 (1986).

[23] C.W.J. Beenakker, H. van Houten, Electronic Properties of Multilayers and Low-Dimensional Semiconductor Structures, Plenum Press, New York 1990, p. 74.

[24] T.J. Thornton, Rep. Prog. Phys. 57, 311 (1994).

[25] S. Datta, Electronic Transport in Mesoscopic Systems, University Press, Cambridge 1995.

[26] Y. Takagaki, K. Gamo, S. Namba, S. Ishida, S. Takaoka, K. Murase, K. Ishibashi, Y. Aoyagi, Solid State Commun. 68, 1051 (1988).

[27] G. Meier, T. Matsuyama, Appl. Phys. Lett. 76, 1315 (2001).

[28] G. Meier, D. Grundler, in: Festkörperprobleme - Advances in Solid State Physics, Ed. B. Kramer, Vol. 40, Vieweg, Braunschweig 2000, p. 295.

[29] G. Meier, M. Halverscheid, T. Matsuyama, U. Merkt, J. Appl. Phys. 89, 7469 (2001).

[30] T.M. Hengstmann, D. Grundler, N. Klockmann, H. Rolff, D. Heitmann, submitted to IEEE Trans. Magn.

[31] K.S. Novoselov, A.K. Geim, S.V. Dubonos, Y.G. Cornelissens, F.M. Peeters, J.C. Maan, Phys. Rev. B 65, 233312 (2002).

[32] Micromagnetic simulations have been performed by $H$. Rolff using the commercially available program MagFEM3D, provided by K. Ramstöck, www.ramstock.de

[33] H.X. Tang, F.G. Monzon, R. Lifshitz, M.C. Cross, M.L. Roukes, Phys. Rev. B 61, 4437 (2000).

[34] G. Schmidt, D. Ferrand, L.W. Molenkamp, A.T. Filip, B.J. van Wees, Phys. Rev. $B$ 62, R4790 (2000).

[35] E.I. Rashba, Phys. Rev. B 62, R16267 (2000).

[36] M. Johnson, IEEE Trans. Magn. 36, 16267 (2000).

[37] A. Fert, H. Jaffres, Phys. Rev. B 64, 184420 (2001).

[38] H.J. Zhu, M. Ramsteiner, H. Kostial, M. Wassermeier, H.-P. Schönherr, K.H. Ploog, Phys. Rev. Lett. 87, 016601 (2001).

[39] P.R. Hammar, M. Johnson, Appl. Phys. Lett. 79, 2591 (2001).

[40] A.T. Hanbicki, B.T. Jonker, G. Itskos, G. Kioseoglon, A. Petrou, Appl. Phys. Lett. 80, $1240(2002)$.

[41] V.F. Motsnyi, J. De Boeck, J. Das, W. Van Roy, G. Borghs, E. Goovaerts, V.I. Safarov, Appl. Phys. Lett. 81, 265 (2002).

[42] Magnetic Properties of Metals: d-Element, Alloys, and Compounds, Ed. H.P.J. Wijn, Springer, Heidelberg 1991, p. 17.

[43] G.E. Blonder, M. Tinkham, Phys. Rev. B 27, 112 (1983).

[44] O. Wunnicke, Ph. Mavropoulos, R. Zeller, P.H. Dederichs, D. Grundler, Phys. Rev. B 65, R241306 (2002).

[45] M. Zwierzycki, K. Xia, P.J. Kelly, G.E.W. Bauer, I. Turek, cond-mat/0204422, 2002.

[46] Ph. Mavropoulos, O. Wunnicke, P.H. Dederichs, Phys. Rev. B 66, 024416 (2002). 